\title{
Surfactant Supplementation in the Preterm Rabbit: Effects of Applied Volume on Compliance and Survival
}

\author{
I. LEROY METCALFE, ROSMARIE BURGOYNE, AND GORAN ENHORNING ${ }^{(25)}$ \\ Institute of Medical Science and Department of Obstetrics and Gynaecology, University of Toronto \\ Toronto, Ontario, Canada
}

\section{Summary}

Preterm rabbit neonates, delivered on the 27th day of gestation, were treated before the first breath with a tracheal instillation of ESA, a suspension of lipids extracted from pulmonary surfactant. Lung compliance development and neonatal survival were studied after treatment to determine the effect of suspension volume and concentration. Compliance development was not dependent on either delivery volume or suspension concentration, but rather on the quantity of phospholipids instilled. Indices of improved expansion and stability demonstrated a dose response relationship up to $1.1 \mathrm{mg}$ phospholipid per $\mathrm{g}$ lung. Larger doses neither improved nor detracted from the response. The ability of the neonates to survive and adapt to an air breathing environment was influenced by the instillation volume. Viability was reduced when the treatment volume exceeded $16 \%$ of the functional residual capacity.

\section{Speculation}

Prophylactic surfactant supplementation therapy may be useful in preventing the development of neonatal respiratory distress syndrome. Surfactant extract may prove acceptable for both prophylaxis and treatment of established respiratory distress syndrome.

A deficiency of surface active lipids in the airways of preterm infants may lead to development of the respiratory distress syndrome (RDS) $(2,6)$. It has been demonstrated in animal models that instillation of surfactant into the upper airways of the preterm neonate before the first breath will facilitate airway expansion, promote stability, improve gas exchange, and improve survival (for review, see 21). More recently, postnatal administration of surfactant has been used to treat advanced cases of RDS in human infants $(7,24)$.

Using the preterm rabbit as a model, we have previously demonstrated (18) the equivalence to natural surfactant (NSA) of a low protein $(<0.01 \%)$ preparation of extracted lipids (ESA). We compared the ability of the two preparations, NSA and ESA, to increase compliance and lung stability and to prolong neonatal survival. We also examined the effect of using a fixed volume, but varying the concentration of the two surface active materials applied. The results demonstrated that with increasing surfactant concentration, total lung capacity, and residual volume at end expiration improved progressively, but reached a plateau at concentrations of $50 \mathrm{mg} / \mathrm{ml}$. We hypothesized that the observed response might not only be related to the concentration but also to the volume of the applied suspension. A change in volume could affect the airway distribution of the preparation. We now report on the effect of varying the volume of the ESA deposited in the trachea before lung expansion.

\section{MATERIALS AND METHODS}

The fetuses from 42 New Zealand white rabbits were examined within $2 \mathrm{~h}$ of the midpoint of their 27 th day of gestation. Normal gestation time for this species is 31 days, with changes in compliance and other indices of pulmonary maturation occurring late on the 28 th or during the 29 th day of pregnancy $(9,14,19)$. Suspensions of lipids extracted from surfactant were prepared as previously described in detail $(17,18)$. Briefly, after procedures to eliminate protein, a known quantity of the lipids in chloroform was dried with a flow of nitrogen on the sides of a test tube and then resuspended in a predetermined volume of isotonic media of $150 \mathrm{mM} \mathrm{NaCl}$ and $5 \mathrm{mM} \mathrm{CaCl} 2$ using a sonic probe in a small test tube surrounded by ice water. The sonicator was set at $100 \mathrm{~W}$ for $1 \mathrm{~min}$. The surfactant thus prepared could be stored in the refrigerator for a few days, but immediately before administration the surface activity of each sample was evaluated using the pulsating bubble technique (5). A sample was considered acceptable if it demonstrated ability to form a film in the air-liquid interface which gave, within $15 \mathrm{sec}$, surface tensions of less than $30 \mathrm{mN} / \mathrm{m}$ when expanded and $0 \mathrm{mN} / \mathrm{m}$ with $50 \%$ compression of the surface area.

Pulmonary compliance. In our previous study we evaluated compliance after deposition of ESA at varying concentrations in a fixed volume $(25 \mu \mathrm{l})$. In this study we used different volumes either at a fixed concentration $(30 \mathrm{mg} / \mathrm{ml})$ or with a fixed amount of phospholipids $(750 \mu \mathrm{g})$. We knew from pilot studies that the fixed concentration we decided on, $30 \mathrm{mg} / \mathrm{ml}$, would improve aeration, but would not result in a maximally expanded lung.

The doe was sacrificed with a lethal dose of sodium pentobarbital $(25 \mathrm{mg} / \mathrm{ml})$. The uterus was excised and $0.5 \mathrm{ml}$ of sodium pentothal was administered intracranially to the fetuses while still in the amniotic sac. Starting from the left horn, the fetuses were numbered to the vagina, and in the right horn in the opposite direction. They were then delivered through uterine incisions and weighed, taking into account the weight of the anesthetic. A polyethylene tube (PE 50) was inserted into the trachea of each fetus. The tubing contained the ESA suspension or, in the case of the control group, the catheter was empty. Control neonates were not given a similar volume of saline for two reasons. First, we wished to compare the treatment response to a normal delivery situation when the neonate is initially presented with an air environment. Second, in a previous study examining this situation (4) we showed that instillation of $25 \mu \mathrm{l}$ of saline to the control group gave no positive response. With larger applied volumes, there might have been a detrimental effect on control compliance, in which case our comparison to the treatment would have been biased.

For the portion of the study investigating the effect of applying ESA in a fixed concentration, 187 fetuses from 23 does were used. The volumes were administered in the following sequential order 
to the littermates: $8.3 \mu \mathrm{l}(n=24), 12.5 \mu \mathrm{l}(n=23), 20.8 \mu \mathrm{l}(n=23)$, $25 \mu \mathrm{l}(n=24), 41.7 \mu \mathrm{l}(n=23), 62.5 \mu \mathrm{l}(n=23)$, and $83.3 \mu \mathrm{l}(n=$ 24). These volumes were chosen so that the amounts of phospholipid instilled into the airways would be equivalent to those used in the previous study when the volume was $25 \mu$ l. A new litter would begin where the previous one left off. All litters contained at least one control $(n=23)$, the position of which was randomly assigned. We determined the total lung capacity (TLC) developed, residual volume (RV) at end expiration, and the air volume remaining in the lungs at a deflation pressure of $10 \mathrm{~cm}$ water as a percentage of the TLC (VD10/TLC $\times 100)$.

Eighty-seven fetuses from 19 does were examined using a fixed quantity of phospholipids $(750 \mu \mathrm{g})$ in volumes of $12.5,25,50,75$, and $100 \mu \mathrm{l}$. We evaluated the same three characteristics (TLC, RV, VD 10/TLC $\times 100$ ) as in the previous part of the study.

Littermates were assessed for compliance simultaneously as described previously (4). The tracheal catheters were connected to a horizontal array of glass tubings containing $2 \mathrm{ml}$ of air. Connected with the other end of the tubings was an open reservoir with stained water, the surface of which was moved so as to give pressures of $0,10,20,30$, and $35 \mathrm{~cm}$ water during inflation, and $30,20,10$, and $0 \mathrm{~cm}$ water during deflation. Each pressure was maintained for $15 \mathrm{sec}$. As air moved from the glass tubings into the lungs, it was replaced with stained water, the volume of which was recorded photographically against a background scale. Two cycles were determined for each group of littermates. The pressurevolume loops were obtained at room temperature.

Survival assessment. Duration of survival was assessed in 84 neonates from 18 litters. Under local anesthesia, a lethal intracranial dose of sodium pentobarbital was administered to the mother. This method of euthanasia minimized the risk of anesthetic transfer to the fetuses. The uterus was exposed and the vessels clamped with a large hemostat. The pups were delivered through uterine incisions while the thorax was compressed digitally to inhibit inspiratory movements. The tracheae were exposed and catheterized with PE 50 tubing containing $1250 \mu \mathrm{g}$ of ESA in a volume of $12.5,25,75$, or $100 \mu \mathrm{l}$. Only the first four or five neonates were used from each litter, since those remaining in utero while the surgery took place became asphyxiated. After catheterization, the thoracic compression was released by rubbing the dorsal skin, and the animals were stimulated to breathe and to aspirate the ESA. Only neonates responding to the stimulation and attempting inspiration were used in the study. When the ESA had been drawn into the airways, the catheter was cut to minimize the dead space, but about $1.5 \mathrm{~cm}$ of tubing was left exposed. Each neonate was transferred to an incubator at $37^{\circ} \mathrm{C}$, supplied with $40 \%$ humidified oxygen. Breathing was monitored every $15 \mathrm{~min}$ for a $3-\mathrm{h}$ period. Our definition of survival was a respiration rate of at least 6 breaths per min. The littermates were stimulated by intermittently shaking the incubator.

The fetuses remaining in utero from the 18 does, plus one extra litter, were used for the experiments in which the phospholipids were supplied in a fixed quantity $(750 \mu \mathrm{g})$, but in a variable volume. Figure 1 provides a graphic display of the three principles for administering the surface active phospholipids: in a fixed amount $(750 \mu \mathrm{g})$, a fixed concentration $(30 \mathrm{mg} / \mathrm{ml})$, or in a fixed volume $(25 \mu \mathrm{l})$.

\section{RESULTS}

Lung compliance. The pressure-volume loops from the fetuses receiving the different volumes of ESA $(30 \mathrm{mg} / \mathrm{ml})$, when compared with those of the control group, demonstrated significantly greater compliance (Fig. 2). At pressures more than $20 \mathrm{~cm}$ water, the volumes of air entering the lungs and retained during deflation were larger in both the first and second expansion cycles. Using successively larger volumes of the ESA with a fixed concentration produced progressively wider hysteresis loops. This was true up to $41.7 \mu \mathrm{l}$, but with larger volumes of ESA (62.5 and $83.3 \mu \mathrm{l})$ there was no further improvement.

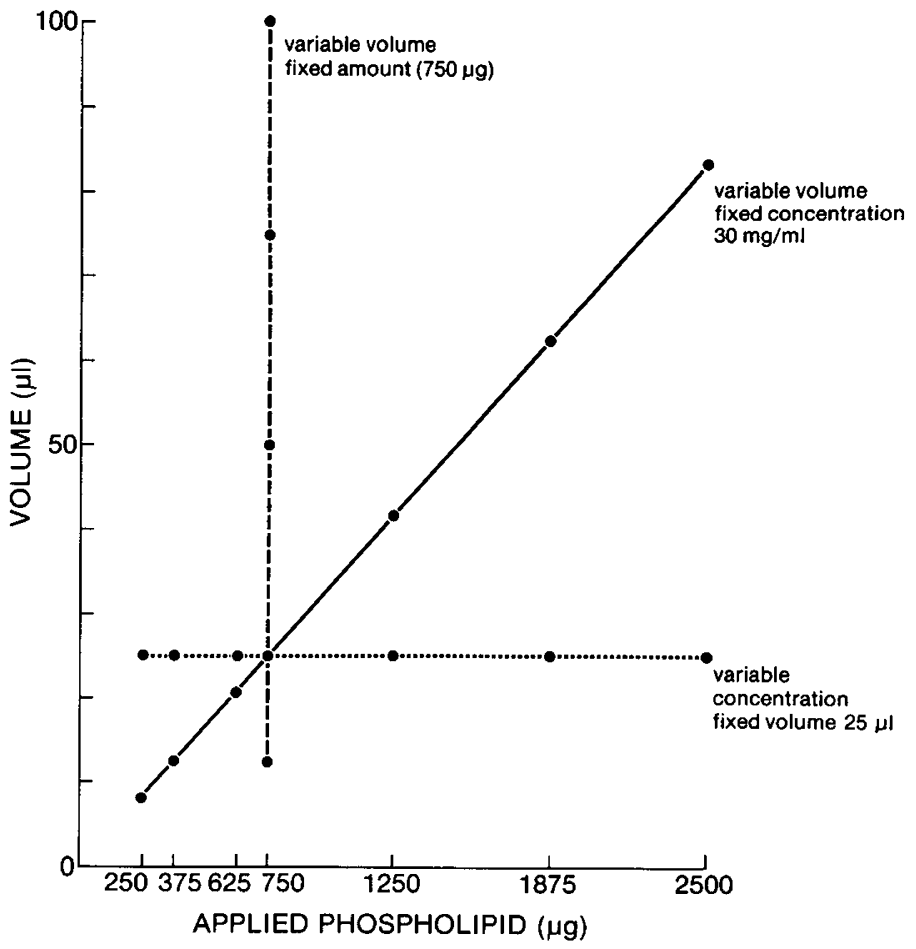

Fig. 1. Graph of suspension volume versus the amount of phospholipids introduced into the airways of preterm 27 th-day rabbit fetuses for each of the three studies, varying volume and concentration.

\section{1st cycle PV loop}

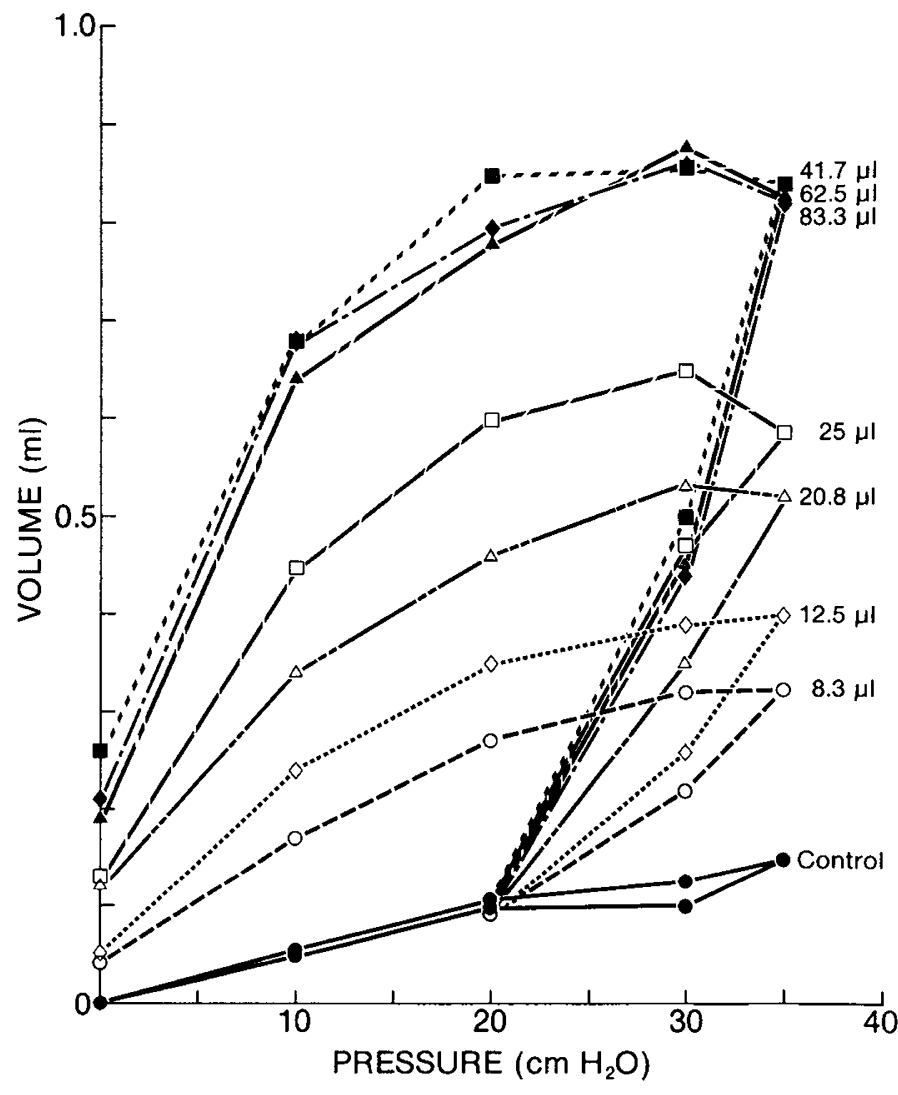

Fig. 2. Pressure-volume loops showing mean volumes of air in the lungs of fetuses for the first inflation-deflation cycle after treatment with different volumes of extracted lipids $(30 \mathrm{mg} / \mathrm{ml})$. Increasing the volume and amount of surface active lipid resulted in increased hysteresis up to $41.7 \mu 1$. 
Figure 3 shows the means with SE for the three indices specifically evaluated: TLC, RV, and VD10/TLC $\times 100$. Instillation of $41.7 \mu \mathrm{l}$ gave the optimal response, whereas larger volumes of the $30 \mathrm{mg} / \mathrm{ml} \mathrm{ESA}$ suspension neither enhanced nor detracted from the response, and a plateau resulted.

A comparison of the mean volumes of air in the lungs with SE during the first and second "breathing" cycles is shown in Figure 4 for the seven different ESA amounts tested. The results obtained from a previous study in which volume was fixed, but concentration was varied, are compared with those of the present study when the concentration was fixed and the volume varied. All groups were equivalent with regard to mean body weight. In the comparison of the two studies, the application of equivalent amounts of phospholipid resulted in the same degree of expansion and stability regardless of the differences in volume or concentration. The compliance improved from the first breath to the second and with increasing amounts of ESA up to $1250 \mu \mathrm{g}$. The introduction of larger amounts of lipid into the fetal airways did not improve the response.

In the study with fixed quantity $(750 \mu \mathrm{g})$ but variable volume, the increase from 12.5-100 $\mu$ l gave a linear response with slope zero for each of the three respiratory parameters: TLC, RV, and VD10/TLC $\times 100$ (Fig. 5).

Survival assessment. Mean body weight of the control and treatment groups was equivalent. All treatment groups demonstrated significantly longer $(P<0.001)$ mean survival than did the control animals. The latter, receiving no tracheal instillation, were unable to maintain regular inspiratory efforts and all had ceased breathing within $30 \mathrm{~min}$ (Fig. 6). The majority of the newborns receiving ESA, even in the smallest volume, became pink and

VARIABLE VOLUME FIXED LIPID CONCENTRATION $(30 \mathrm{mg} / \mathrm{ml})$

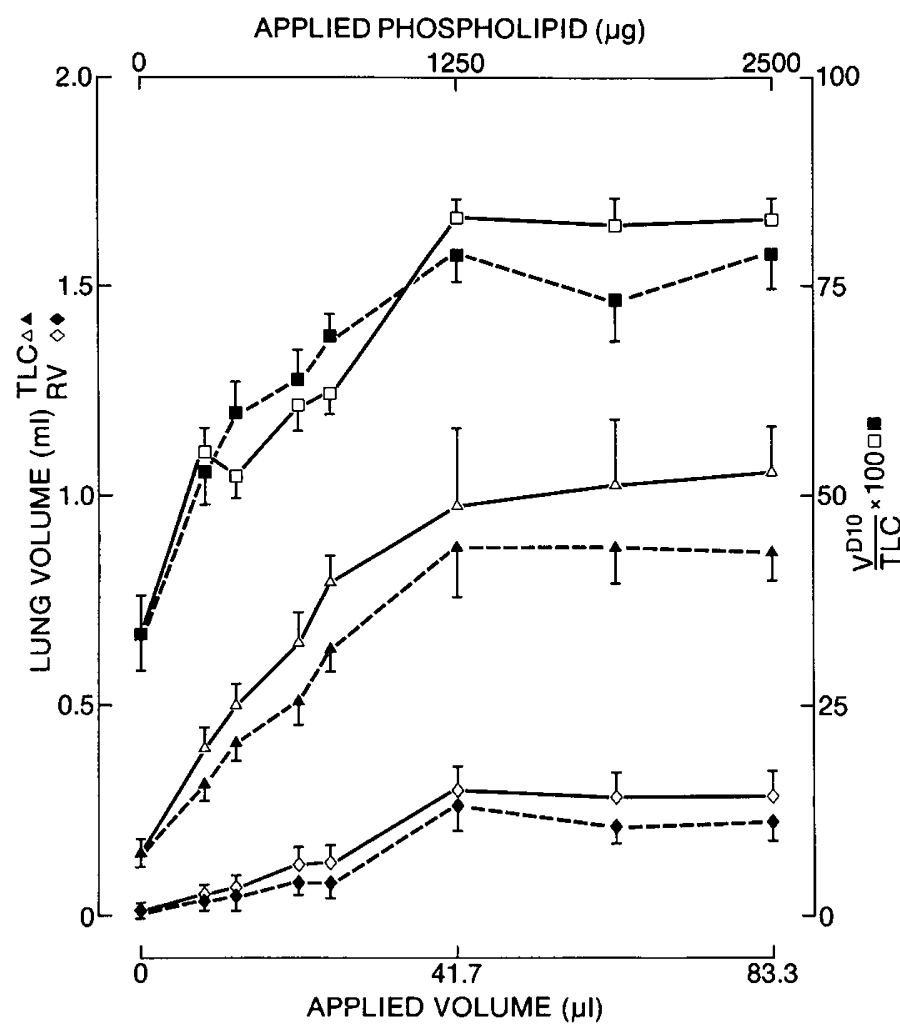

Fig. 3. The effect of varying the applied volume of extracted lipids with a fixed concentration on first (closed symbols) and second (open symbols) lung expansions. Residual volume (RV) $(\diamond \diamond)$, total lung capacity (TLC) $(\Delta \triangle)$ and volume at deflation pressure $10 \mathrm{~cm}$ water as a $\%$ of TLC

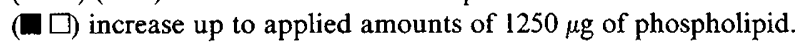

lively within $15 \mathrm{~min}$ following active inspiration of the material. The distribution of rabbit pups surviving the entire time course of the experiment was as follows: $85 \%$ for $12.5 \mu \mathrm{l}$ treatment group, $85 \%$ for the $25 \mu \mathrm{l}$ group, $93 \%$ for the $50 \mu \mathrm{l}$ group, $71 \%$ for the 75 $\mu \mathrm{l}$ group, and $64 \%$ for the $100 \mu \mathrm{l}$ volume. There were some unexpected responses exhibited by several neonates in the two treatment groups receiving the largest instilled volumes (75 to 100 $\mu 1)$. We had set a criteria of at least six respirations per minute as being an index of fetal viability. Two neonates in the $75 \mu \mathrm{l}$ treatment group and four in the $100 \mu \mathrm{l}$ group surprisingly initiated regular breathing after having expired according to our definition. In each of these six cases, the neonates were apneic and cyanotic, then suddenly initiated normal breathing at times ranging between 30-90 minutes after the tracheal deposition. After their recovery, the neonates were monitored for $3 \mathrm{~h}$ and all survived that observation period.

\section{DISCUSSION}

Our initial hypothesis was that the volume in which the ESA was applied might be an important factor in the neonatal response to surfactant supplementation. The results of the survival studies were in accordance with this hypothesis, but the compliance study results were not. Surface active lipids did indeed induce changes in compliance which, however, reflected the amount of lipids instilled and not the volume in which they were administered. Generally, volume was insignificant, but when it was extremely small as with the highest concentration in the experiments with a fixed amount of phospholipids $(750 \mu \mathrm{g})$, there was a decreased response, albeit without statistical significance, on the three indices studied: TLC, RV, and VD10/TLC $\times 100$. As an explanation, one might point to the higher viscosity of the most concentrated surfactant preparation, or to the fact that material was lost by adhering to the wall of the polyethylene tube through which it had been instilled into the trachea. By rinsing the tube with chloroform and analyzing for phosphorus, it was found that the loss of material was greatest with the highest concentrations and could then be $5-15 \%$. In the experiments with fixed ESA concentration, obviously more surfactant was instilled with the two largest volumes, and yet this did not induce any further improvement. Probably the amount necessary to develop the maximal change in compliance was less than what was contained in these two larger volumes, and the lungs could only be expanded to the limit of their capacity.

The survival study data indicated that the volume of instilled fluid did affect the establishment of air breathing. In the dead animal, it would hardly make a noticeable difference if the lungs were partly expanded with a liquid rather than air, but in the living animal such a replacement would reduce gas exchange and could be a cause of neonatal asphyxia. The higher mortality and the long periods of apnea observed with instillation of the largest volume of surfactant could thus be explained. It was not until the fluid instilled into the airways had been partly absorbed that adequate gas exchange could be established. Normally, after birth and initial aeration, pulmonary blood flow increases $(3,22)$ and airway liquid is quickly absorbed $(1,10)$. If full aeration does not occur and the subsequent perfusion changes are compromised, clearance of the pulmonary fluid may not proceed as rapidly. It is known that the preterm rabbit fetus can maintain cardiac activity for prolonged periods even with no oxygen supply (8). We hypothesize therefore that with the instillation of larger volumes some neonates were unable to clear the fluid and did not establish aeration or regular breathing patterns. In some cases the absorption of fluid continued and if a sufficient volume was cleared and if the neonates could still maintain the ability to initiate respiratory efforts, they could establish regular respiration. In those instances when the neonates did not initiate breathing within the observation period of $3 \mathrm{~h}$, they probably were unable to handle the excessive volume of liquid and succumbed to anoxia. 

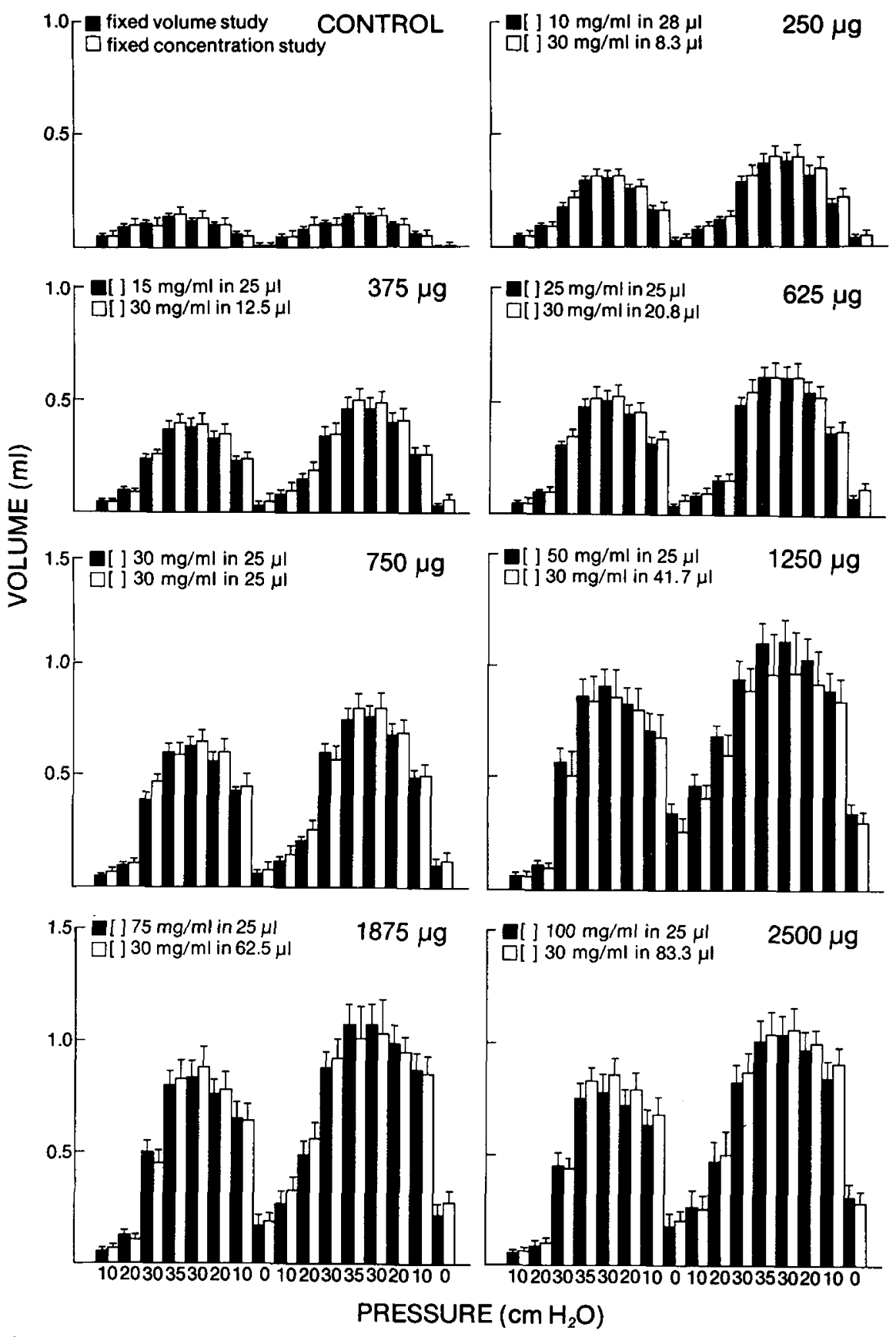

Fig. 4. A comparison of the mean volumes of air present in the airways of neonates treated with equivalent amounts of surface active extracted lipids in different volumes. For any amount of applied lipid there was an equivalency at all pressures regardless of suspension concentration or administered volume.

When surfactant supplementation is considered as a therapy or as prophylaxis, knowledge of the dose response is obviously important. For an optimal effect on aeration and on airway stability, the dose in this study was $1250 \mu \mathrm{g}$ or $0.05 \mathrm{mg}$ of phospholipid per $\mathrm{g}$ body weight. The TLC developed was in the region of $1 \mathrm{ml}$ of air, which is approximately equal to that developed by rabbit neonates delivered 2 days later on the 29 th day of gestation (19). Based on a wet lung weight of $1.1 \mathrm{~g}$ for the rabbit fetus on the 27 th day of gestation (19), the peak compliance response could be obtained with the instillation of $1.1 \mathrm{mg}$ phospholipid per $g$ lung. This value is very close to that reported for adult lung (15) and twice the dosage reported by Ikegami et al. (11) as being necessary to develop optimal compliance in the preterm sheep neonate $(0.56 \mathrm{mg}$ phospholipid/g lung) using natural surfactant. It was interesting that the application of twice the amount required for maximal expansion and stability had no negative effect. Normally, the onset of birth is accompanied by a mechanical release of surfactant from stored inclusion bodies in the type II pneumocyte $(14,16)$, which raises the amount of surfactant to three times adult levels (9). The instillation of excess surfactant may mimic this situation and prolong the duration of the supplementation effect by acting as a reservoir. The introduction of such a reserve supply might be necessary because of the slow accumulation of surfactant in the airways by de novo synthesis after birth (12), which makes the initial maintenance of alveolar stability primarily dependent on surfactant stores. We did not assess the response duration in our study beyond the 3 -h observation time, because we were interested in the immediate benefit of supplementation therapy to neonatal survival. Longer periods of examination have the added complications of nutrient provision and non-treatment-related problems. Jobe et al. (13) have reported a short-term improvement with the administration of natural 
VARIABLE VOLUME FIXED LIPID QUANTITY $(750 \mu \mathrm{g})$

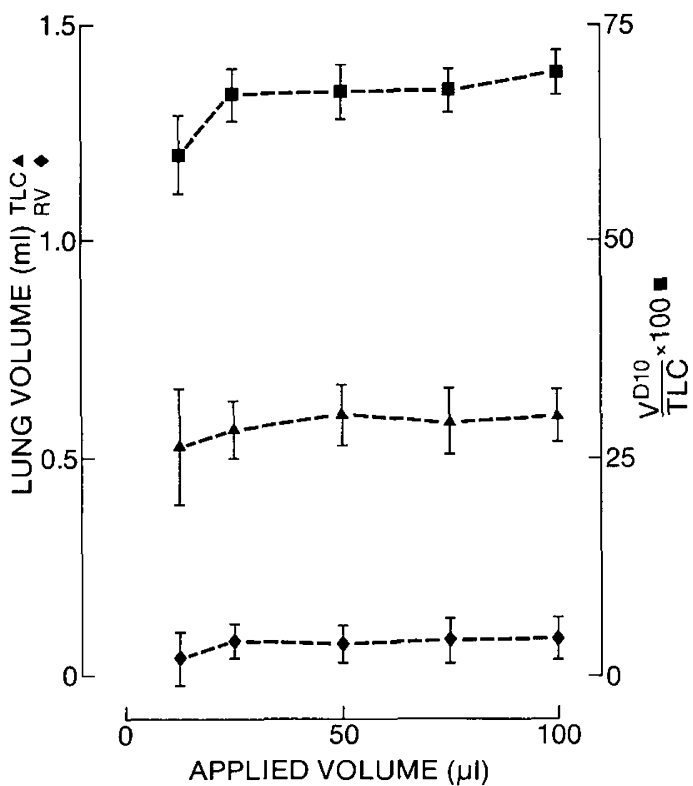

Fig. 5. Graph of the three respiratory indices: Residual volume (RV $(\diamond)$, total lung capacity (TLC $(\Delta)$, and VD10/TLC $\times 100(\square)$ when the amount of applied extracted lipids was fixed and the volume varied. There was no difference in response level for any administered volume.

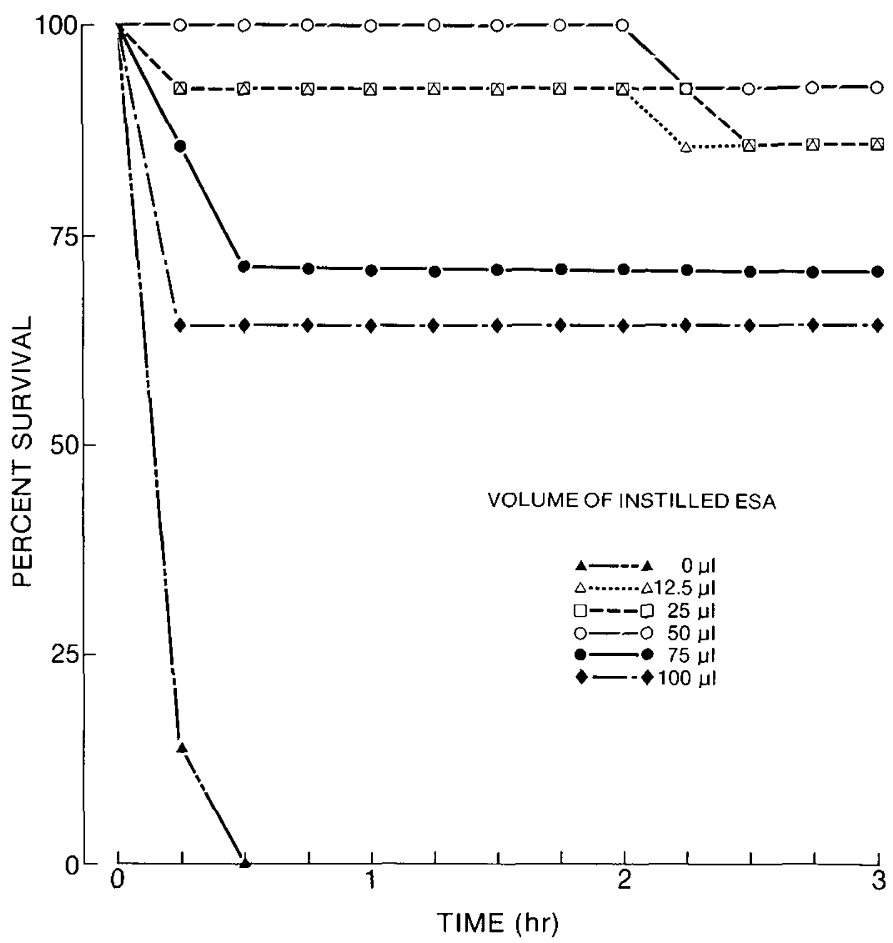

Fig. 6. \% survival distribution of preterm neonates treated with 1250 $\mu \mathrm{g}$ of extracted lipids (ESA) in different volumes for their first breath. Increasing the volume to greater than $16 \%$ of the functional residual capacity reduced the level of survival.

surfactant to preterm fetal lambs. Inasmuch as the surfactant introduced in their study was extremely hypotonic and hemorrhagic lung washes were observed at autopsy, the treatment procedure may have been suboptimal.

In the sheep fetus, the amount of pulmonary fluid is approximately equal to the functional residual capacity (FRC) (23). Based on the assumptions that this is also true for the rabbit model and that the FRC is approximately one-third of the TLC as it is with the adult, then the total FPF volume for our model was in the region of $300 \mu \mathrm{l}$. The load volumes of $12.5,25,50,75$, and $100 \mu \mathrm{l}$, used in the survival study, therefore represented approximately 4 , $8,16,25$, and $33 \%$, respectively, of the volume of FPF present in the airways at birth. Extrapolation of the results suggest that if ESA were applied as a prophylactic supplement to the human neonate, a minimal amount equivalent to $1 \mathrm{mg}$ phospholipid per $\mathrm{g}$ lung should be administered, although larger quantities may prove beneficial in prolonging the response. Based on a $40 \mathrm{~g}$ lung with an FRC of $35 \mathrm{ml}$ (for a $1.5 \mathrm{~kg}$ neonate), this would translate to $40 \mathrm{mg}$ of ESA phospholipids suspended in a volume of 1.5-6 $\mathrm{ml}$ (4-16\% of FRC). Because the amount of fluid in the lung decreases towards term (19), equivalent volumes applied at different times of gestational development (12) may represent different clearance loads for the fluid resorption system.

Surfactant supplementation has been used to treat established RDS, initially by Fujiwara et al. (7) and in North America using our sterilized ESA preparation (24). Both treatment courses used large quantities of phospholipid (150-200 mg) and volumes (8-10 $\mathrm{ml}$ ) in a single applied dose. In both of these studies it is evident that the neonatal lung can tolerate larger amounts than the minimum recommended by this report. The instillation of these larger quantities may have been responsible for the fact that only one dose was required. In our experiments, the volumes were administered before the first breath and the initiation of FPF resorption. The situation in which surfactant is used to treat established RDS may be different, since in that circumstance partial resorption of FPF would have occurred. This may have the effect of increasing the ability of the airways to tolerate larger added volumes. A recent report by Morley et al. (20) describes the successful prophylactic treatment for RDS using a dry synthetic surfactant preparation. One $25 \mathrm{mg}$ dose was blown into the airways of apneic preterm neonates after resuscitation. This delivery method would seem to have the advantage of not introducing a load volume which the lung then has to clear. The results of our study, however, would indicate that the neonatal lung has a great capacity for such clearance. Furthermore, the distribution of a liquid suspension to the terminal airspaces should be more widespread than a powdered lipid which might tend to hydrate and adhere to the sides of the larger airways. Although the techniques of administration were different, the results from our study would suggest that, for an optimal response, a larger quantity of dry surfactant material would be needed than that used by Morley $e t$ al.

\section{REFERENCES AND NOTES}

1. Aherne, W. and Dawkins, M.: The removal of fluid from the pulmonary airways after birth in the rabbit, and the effect on this of prematurity and pre-nata hypoxia. Biol. Neonate, 7: 214 (1964).

2. Avery, M. E. and Mead, J.: Surface properties in relation to atelectasis and hyaline membrane disease. Am. J. Dis. Child., 97: 517 (1959).

3. Dawes, G. S., Mott, J. C., Widdecombe, J. G., and Wyatt, D. G.: Changes in the lungs of the new-born lamb. J. Physiol. (Lond), I21: 141 (1953).

4. Enhorning, G. and Robertson, B.: Lung expansion in the premature rabbit fetus after tracheal deposition of surfactant. Pediatrics, 50: 58 (1972).

5. Enhorning, G.: A pulsating bubble technique for evaluating pulmonary surfactant. J. Appl. Physiol., 43: 198 (1977).

6. Farrell, P. M. and Avery, M. E.: Hyaline membrane disease. Am. Rev. Resp. Dis., 111: 657 (1975).

7. Fujiwara, T., Chida, S., Watabi, Y., Maeta, H., Morita, T., and Abe, T.: Artificial surfactant therapy in hyaline membrane disease. Lancet, $l: 55$ (1980).

8. Gelli, M. G., Enhorning, G., Hultman, E., and Bergstrom, J.: Glucose infusion in the pregnant rabbit and its effect on glycogen content and activity of foetal heart under anoxia. Acta Pediatr. Scand., 57: 209 (1968).

9. Gluck, L., Motoyama, E. K., Smits, H. L., and Kulovich, M. V.: The biochemical development of surface activity in mammalian lung. I. The surface active phospholipids: The separation and distribution of surface active lecithin in the lung of the developing rabbit fetus. Pediatr. Res., 1: 237 (1967).

10. Humphreys, P. W., Normand, I. C. S., Reynolds, E. O. R., and Strang, L. B. Pulmonary lymph flow and the uptake of liquid from the liquids from the lungs of the lamb at the start of breathing. J. Physiol. (Lond), 193: 1 (1967).

11. Ikegami, M., Adams, F. H., Towers, B., and Osher, A. B.: The quantity of natural surfactant necessary to prevent the respiratory distress syndrome in premature 
lambs. Pediatr. Res., 14: 1082 (1980).

12. Jobe, A. and Gluck, L.: The labeling of lung phosphatidylcholine in premature rabbits. Pediatr. Res., 13: 635 (1979).

13. Jobe, A., Ikegami, M., Glatz, T., Yoshida, Y., Diakomanolis, E., and Padbury, J.: Duration and characteristics of treatment of premature lambs with natural surfactant. J. Clin. Invest., 67: 370 (1981).

14. Kikkawa, Y., Motoyama, E. K., and Gluck, L.: Study of the lungs of fetal and newborn rabbits: Morphologic, biochemical, and surface physical development. Am. J. Pathol., 52: 177 (1968)

15. King, R. J. and Clements, J. A.: Surface active materials from dog lung. II Composition and physiological correlations. Am. J. Physiol., 223: 715 (1972).

16. Lawson, E. E., Birdwell, R. L., Huang, P. S., and Taeusch, H. W.: Augmentation of pulmonary surfactant secretion by lung expansion at birth. Pediatr. Res. 13: 611 (1979).

17. Metcalfe, I. L., Enhorning, G., and Possmayer, F.: Pulmonary surfactant-associated proteins: Their role in the expression of surface activity. J. Appl. Physiol., 49: 34 (1980).

18. Metcalfe, I. L., Pototschnik, R., Burgoyne, R., and Enhorning, G.: Lung expansion and survival in rabbit neonates treated with surfactant extract. (Submitted to J. Appl. Physiol.)

Copyright (C) 1982 International Pediatric Research Foundation, Inc. $0031-3998 / 82 / 1610-0834 \$ 02.00 / 0$
19. Metcalfe, I. L., Burgoyne, R., and Enhorning, G.: Surfactant-related parameters in the maturing rabbit fetus. (Unpublished observations.)

20. Morley, C. J., Bangham, A. D., Miller, N., and Davis, J. A.: Dry artificial lung surfactant and its effect on very premature babies. Lancet, $1: 64$ (1981)

21. Robertson, B.: Surfactant substitution: Experimental models and clinical applications. Lung, 158: 57 (1980)

22. Rudolph, A. M.: The changes in the circulation after birth. Circulation, $41: 343$ (1970).

23. Scarpelli, E. M., Condorelli, S., and Cosmi, E. V.: Lamb fetal pulmonary fluid. I. Validation and significance of method for determination of volume and volume change. Pediatr. Res., 9: 190 (1975).

24. Smyth, J. A., Metcalfe, I. L., Duffty, P., Enhorning, G., Possmayer, F., Olley, P. M., and Bryan, M. H.: Surfactant therapy in hyaline membrane disease. Pediatr. Res., 15: 681 (1981).

25. Requests for reprints should be addressed to: Goran Enhorning, M.D., Toronto Western Hospital, 399 Bathurst Street, Toronto, Ontario, Canada M5T 2S8.

26. This research was supported by the Medical Research Council of Canada, Grant No. MT-4497.

27. Received for publication June 11, 1981.

28. Accepted for publication March 12, 1982. 